\title{
Synergien zwischen nicht-digitalen und digitalen Geschäftsmodellen in Unternehmen: Möglichkeiten und Handlungsempfehlungen
}

\author{
Jonas Toutaoui $(\mathbb{D}) \cdot$ Lea Müller • Alexander Benlian
}

Eingegangen: 1. Oktober 2020 / Angenommen: 16. Dezember 2020 / Online publiziert: 5. Januar 2021

(C) Der/die Autor(en) 2021

Zusammenfassung Führungskräfte erkennen zunehmend, wie wichtig digitale Geschäftsmodelle sind, um die digitale Transformation in Unternehmen mit bestehenden nicht-digitalen Geschäftsmodellen erfolgreich umzusetzen. Dennoch fehlt oft das Bewusstsein für Synergien, die durch koexistente digitale und nicht-digitale Geschäftsmodelle innerhalb desselben Unternehmens möglich sind. Unsere Studie hebt digitale Plattformen als entscheidende Mechanismen hervor, die es etablierten Unternehmen ermöglichen, diese Synergien zu nutzen. Wir identifizieren sechs Arten von Synergien zwischen nicht-digitalen und digitalen Geschäftsmodellen, die etablierten Unternehmen helfen, einen Mehrwert im Vergleich zu einer bloßen Koexistenz dieser Geschäftsmodelle zu schaffen. Darüber hinaus bilden wir diese Synergien auf die einzigartigen Merkmale und Dynamiken digitaler Plattformen ab. Der Artikel schließt mit Empfehlungen zur Freisetzung dieser Synergien entlang der verschiedenen Phasen der Geschäftsmodellentwicklung.

Schlüsselwörter Digitale Geschäftsmodelle · Digitale Transformation · Synergien · Plattform-Dynamik · Netzwerkeffekte $\cdot$ Mehrseitige Plattformen

J. Toutaoui $(\bowtie) \cdot$ L. Müller $\cdot$ A. Benlian Fachgebiet Information Systems \& E-Services, Technische Universität Darmstadt, Hochschulstr. 1, 64289 Darmstadt, Deutschland E-Mail: toutaoui@ise.tu-darmstadt.de 


\title{
Synergies between Non-Digital and Digital Business Models: Opportunities and Recommendations
}

\begin{abstract}
Organizational leaders increasingly know of the importance of digital business models to master the digital transformation journey in established companies, yet the awareness for synergies created by concurrent digital and non-digital business models within the same company is often lacking. Our study identifies digital platforms as critical linchpins for established companies to leverage synergies. We uncover six types of synergies between non-digital and digital business models that help established companies exploit added value compared to a mere coexistence of these business models. We additionally map these synergies to unique digital platform characteristics and dynamics. The article concludes with recommendations to unlock these synergies along the different phases of business model development.
\end{abstract}

Keywords Digital business models - Digital transformation - Synergies $\cdot$ Platform dynamics $\cdot$ Network effects $\cdot$ Multisided platforms

\section{Einführung}

Die Digitalisierung verändert die Anforderungen der Kunden, schafft neue Produkte, durchbricht traditionelle Märkte und fordert etablierte Unternehmen heraus. Als Reaktion darauf entwickeln ebendiese Unternehmen vielfältige Digital-Strategien mit dem Ziel, ihre vorhandenen Stärken und die neuen Möglichkeiten zu nutzen (Sebastian et al. 2017; Hess et al. 2016). Dazu gehören die Digitalisierung interner Prozesse zur Steigerung der Mitarbeiterproduktivität oder eine digitale Kundenschnittstelle zur Erfüllung von Kundenerwartungen, aber auch gänzlich neue digitale Geschäftsmodelle. Tatsächlich setzen fast neun von zehn etablierten Unternehmen digitale Geschäftsmodelle in Form von digitalen Plattformen um (McKinsey 2019) - softwarebasierte digitale Umgebungen, die durch die Erleichterung direkter Interaktionen zwischen Teilnehmergruppen wie Kunden oder Komplementären einen Mehrwert schaffen (Hagiu und Wright 2015).

Die Implementierung neuer digitaler Geschäftsmodelle bedeutet nicht, dass das bestehende Geschäftsmodell verworfen wird. Vielmehr finden sich in Unternehmen nun zwei koexistente Geschäftsmodelle, ein nicht-digitales und etabliertes sowie ein digitales und neues (Toutaoui und Benlian 2020). Diese werden bisher oft recht unabhängig voneinander gesteuert. Synergiepotenziale werden dabei nicht systematisch berücksichtigt oder gar gehoben. Der gleichzeitige Betrieb von Geschäftsmodellen eröffnet jedoch Synergiemöglichkeiten. Synergien können, wenn sie klug gehandhabt werden, ein Segen sein und zusätzlichen Wert schaffen (Goold und Campbell 1998; Martin und Eisenhardt 2001). Für Führungskräfte ist es daher von entscheidender Bedeutung, sich aller Quellen von Synergien und der Möglichkeiten, sie zu managen, bewusst zu sein. Dies gilt insbesondere für Synergien, die durch die digitale Transformation ermöglicht werden. Um mit der großen Zahl von auf den Markt drängenden Start-ups konkurrieren zu können, müssen etablierte Unternehmen die Ressourcen und Erfahrungen aus dem etablierten Geschäftsmodell nutzen. 
Ein Grund dafür, dass einige Unternehmen mit ihrer digitalen Transformation nicht erfolgreich sind, kann die fehlende Nutzung von Synergien zwischen dem digitalen und dem nicht-digitalen Geschäftsmodell sein. Wir stellen daher die folgende Forschungsfrage:

Welche Synergien bestehen zwischen einem neuen zusätzlichen digitalen Geschäftsmodell und dem etablierten nicht-digitalen Geschäftsmodell innerhalb desselben Unternehmens und wie können diese genutzt werden?

Zur Beantwortung dieser Forschungsfrage wurde eine qualitative, interpretative Multi-Fallstudie durchgeführt. Wir haben 16 Entscheidungsträger aus acht verschiedenen Unternehmen unterschiedlicher Branchen und Größen interviewt. Darüber hinaus wurden öffentliche und interne Sekundärdaten dieser Firmen analysiert. Jede hat in der Vergangenheit ein neues, zusätzliches digitales Geschäftsmodell aufgebaut, meist in Form einer digitalen Plattform. In diesem Beitrag gehen wir auf Charakteristika und Dynamiken plattformbasierter digitaler Geschäftsmodelle ein. Dann erläutern wir Synergiemöglichkeiten zwischen digitalen und nicht-digitalen Geschäftsmodellen innerhalb etablierter Unternehmen. Wir enden mit Handlungsempfehlungen für Führungskräfte etablierter Unternehmen, die bereit sind, Synergien zwischen digitalen und nicht-digitalen Geschäftsmodellen zu nutzen.

\section{Plattformbasierte digitale Geschäftsmodelle und Plattform- Dynamiken}

Seit den 1990er-Jahren beschäftigen sich die Wissenschaft und die Praxis mit Geschäftsmodellen und das Forschungsinteresse hat stetig zugenommen (Zott et al. 2011). Teece (2010) definiert ein Geschäftsmodell als ein konzeptionelles Modell eines Unternehmens, das beschreibt, wie dieses Wert erschafft, diesen Wert an Kunden weitergibt und ihn in Profit umwandelt. Während inzwischen nahezu alle Geschäftsmodelle zumindest in ihrer Infrastruktur und Unterstützungsfunktionen auf IT angewiesen sind, gelten Geschäftsmodelle als digital, wenn darüber hinaus sowohl die Kundenschnittstelle als auch das Produkt an sich digital sind, sodass entlang aller Komponenten der Wertschöpfungskette der IT bzw. neuen Digital-Technologien (z. B. Künstliche Intelligenz, Internet der Dinge) eine Schlüsselrolle zukommt (Steininger 2018). Ein Beispiel ist etwa die Simulation von digitalen Zwillingen in der Cloud durch Software-Unternehmen für Industrie-Kunden (Klostermeier et al. 2018). Somit sind digitale Geschäftsmodelle inzwischen ein wichtiger Punkt auf der Forschungsagenda der Wirtschaftsinformatik (Veit et al. 2014).

Aus den zahlreichen Möglichkeiten, neue Geschäftsmodelle zu kreieren, präferieren viele etablierte Unternehmen, plattformbasierte digitale Geschäftsmodelle einzuführen (McKinsey 2019; Pflaum und Schulz 2018). Dabei handelt es sich zunächst nicht um eine Innovation des bestehenden Geschäftsmodells, sondern um die Schaffung eines gänzlich neuen zusätzlichen Geschäftsmodells. Eine digitale Plattform ist eine grundlegende Softwaretechnologie, die verschiedene Interessengruppen digital zusammenbringt und Interaktionen und Transaktionen ermöglicht, welche ohne sie deutlich komplizierter wären (Hagiu und Wright 2015; Evans und 
Schmalensee 2007). Dabei weisen digitale Plattformen einige einzigartige Merkmale und Dynamiken auf.

Eine der wichtigsten Eigenschaften digitaler Plattformen ist ihre Mehrseitigkeit (Tiwana 2014). Jede Seite ist eine eigene Gruppe von Interessenvertretern, die auf der Plattform mit anderen interagieren. Ohne die Plattform hätten diese Interessengruppen Schwierigkeiten, in Kontakt zu kommen und miteinander zu kommunizieren. Die Mehrseitigkeit schafft daher einen Mehrwert, indem sie diese Interaktion erleichtert. Insbesondere für zweiseitige digitale Plattformen gibt es viele prominente Beispiele, wie etwa eBay oder Etsy, welche Käufer und Verkäufer online miteinander verbinden.

Digitale Plattformen können zudem eine Änderung im Verständnis klassischer Wettbewerbsrollen herbeiführen. $\mathrm{Zu}$ den verschiedenen Rollen in einem PlattformÖkosystem gehören der Plattformanbieter, der die Plattform bereitstellt und betreibt, und die Plattformnutzer, die nach Konsumenten und Komplementären unterschieden werden können (Tiwana 2014; McIntyre und Srinivasan 2017). Konsumenten kaufen oder nutzen das, was auf der Plattform in Form von Produkten, Dienstleistungen oder Informationen angeboten wird. Komplementäre stellen der Plattform diese Produkte, Dienstleistungen oder Informationen zur Verfügung. Je nach Offenheit der Plattform können nicht nur Partner, sondern auch Konkurrenten des etablierten Geschäftsmodells des Plattformanbieters die Rolle des Komplementärs übernehmen und so den Wert der Plattform für alle Beteiligten erhöhen (Ondrus et al. 2015).

Ein weiteres wesentliches Merkmal der digitalen Plattformen sind Netzwerkeffekte. Der Begriff bezieht sind auf den Effekt, den ein zusätzlicher Nutzer auf die bestehenden Nutzer einer Plattform hat (Tiwana 2014; Katz und Shapiro 1994). Netzwerkeffekte beeinflussen die Wertschöpfung eines Nutzers aus der Plattform stark und können über ihren Erfolg oder Misserfolg entscheiden (Cennamo und Santalo 2013). Bei einem positiven Netzwerkeffekt erhöht eine größere Anzahl an Plattformnutzern den Wert der Plattform für bestehende Nutzer. Ebenso werden potenzielle neue Nutzer ermutigt, der Plattform beizutreten. Der Netzwerkeffekt hat zu exponentiellen Wachstumsraten bei Plattformen wie z. B. Facebook oder YouTube geführt.

\section{Geschäftsmodell-Synergien}

Geschäftsmodell-Synergien beschreiben positive Auswirkungen, die entstehen, wenn zwei getrennte Geschäftsmodelle miteinander in Verbindung gebracht werden. Unter Führungskräften sind Synergien meist als ein Hebel zur Kostensenkung bekannt. In der Tat führt die Nutzung gemeinsamer Ressourcen über Geschäftsmodelle hinweg zu sogenannten sub-additiven Kostensynergien und die Geschäftsmodelle profitieren von reduzierten Gesamtkosten (Robins und Wiersema 1995). Synergien sind jedoch nicht auf kostensenkende Effekte beschränkt, sondern können auch zu einer, oftmals schwer quantifizierbaren, Wertsteigerung führen, wenn Geschäftsmodell-Komponenten komplementär sind oder wie Milgrom und Roberts (1995) es ursprünglich formulierten: „mehr von einer Sache zu tun, erhöht den Ertrag, mehr von einer anderen zu tun." Komplementäre Geschäftsmodell-Komponenten 
schaffen somit super-additive Wertsynergien, da die gemeinsame Wertschöpfung größer ist als die Kombination der individuellen Wertschöpfungen der einzelnen Geschäftsmodelle.

In den vergangenen Jahrzehnten hatten viele etablierte Unternehmen bereits zusätzliche Geschäftsmodelle aufgebaut. Diese zusätzlichen Geschäftsmodelle ermöglichten es den Unternehmen jedoch nur, innerhalb desselben Marktes eine Niedrigpreis- und eine Premium-Version eines Produktes anzubieten. Beispiele sind Toyota mit seiner Premium-Marke Lexus oder Nestlé mit seiner Nespresso-Tochtergesellschaft, die Premium-Kaffee anbietet. Diese produktorientierten Geschäftsmodelle unterschieden sich in ihren Komponenten hauptsächlich in ihrer Kostenstruktur (Markides und Charitou 2004). Für weiterführende Wirtschaftsinformatik-Forschung haben vor allem Wiener et al. (2018) und Hoßbach (2015) den Grundstein gelegt, als sie Synergien in Omni-Kanal-Geschäftsmodellen untersuchten (z. B. online/printZeitungen). Während diese Studien wertvolle Erkenntnisse für Forschung und Praxis liefern, unterscheiden sich plattformbasierte Geschäftsmodelle jedoch stärker von etablierten produktorientierten Geschäftsmodellen als nur in ihren Kostenstrukturen oder Kanälen und bieten daher zusätzliches Synergiepotenzial (Pflaum und Schulz 2018; Toutaoui und Benlian 2020).

\section{Methodik}

Um die Nutzung von Synergien zwischen digitalen und nicht-digitalen Geschäftsmodellen zu untersuchen, wurden Unternehmen unterschiedlicher Branchen und Größen ausgewählt. Dies erhöht auch die Zuverlässigkeit und Validität der Ergebnisse. Zudem ermöglicht unser Ansatz die Untersuchung von Synergiepotenzialen für verschiedene Branchen und Größen sowie für verschiedene etablierte und digitale Geschäftsmodelle, um Muster in verschiedenen Fällen zu finden (Yin 2003). Alle Unternehmen erfüllen zwei Kriterien: Erstens muss das Unternehmen mit seinem etablierten nicht-digitalen Geschäftsmodell eine starke Marktposition haben. Dieses Kriterium schließt auch rein digitale Unternehmen (z.B. Amazon) aus. Zweitens muss das neue digitale Geschäftsmodell einen gewissen Reifegrad aufweisen (Erfolg bei ersten Kunden), um genügend Informationen liefern zu können. Es wurden semi-strukturierte Interviews mit Entscheidungsträgern aus acht verschiedenen Unternehmen durchgeführt, ähnlich wie Li et al. (2017) und Hanelt et al. (2017). Darüber hinaus wurden die Daten mit Informationen aus internen und öffentlich zugänglichen Unterlagen angereichert, um unsere Ergebnisse zu triangulieren und ihre Validität weiter zu erhöhen. Nachdem der letzte Fall keine neuartigen Ergebnisse lieferte, wurde die Erhebung beendet. Grundsätzlich haben wir uns bei der Konzeption und Durchführung der Studie an die Prinzipien von Klein und Myers (1999) gehalten. Tab. 1 im Anhang enthält weitere Einzelheiten zu den Unternehmen der Multi-Fallstudie sowie ihren nicht-digitalen und digitalen Geschäftsmodellen.

Etablierten Empfehlungen zur qualitativen Datenanalyse folgend (Miles und $\mathrm{Hu}-$ berman 1994), wurde zunächst eine Fall-interne Analyse durchgeführt, welche zu einem umfassenden Verständnis der jeweiligen Geschäftsmodelle beitrug. Ebenso konnte eine Vielzahl an Synergien zwischen den koexistenten Geschäftsmodellen 
identifiziert werden. Mit Hilfe der darauf folgenden Fall-übergreifenden Analyse konnten diese Synergien gruppiert und Muster herausgearbeitet werden. In jedem Fall wurden Synergien identifiziert, wobei nicht jedes Unternehmen alle gefundenen Synergien aufwies. Die Analysen (d.h. die Codierung) wurden von zwei Codierern unabhängig voneinander durchgeführt, welche für zufällig ausgewählte Teilmengen von Daten sehr ähnliche Ergebnisse fanden.

\section{Ergebnisse}

Unsere Forschung enthüllt sechs Arten von Synergien zwischen digitalen und nichtdigitalen Geschäftsmodellen, von denen beide Geschäftsmodelle in hohem Maße profitieren können. Abb. 1 fasst diese Forschungsergebnisse zusammen. Die ersten beiden Synergien sind besonders hilfreich, um das Wachstum zu beschleunigen, indem Netzwerkeffekte des plattformbasierten digitalen Geschäftsmodells gefördert werden. Die nächsten beiden Synergien bauen auf der einzigartigen Dynamik von Mehrseitigkeit und neuen Rollenkonstellationen in Plattformökosystemen auf. Die letzten beiden Synergien betreffen Wissen, das geteilt und wiederverwendet werden kann, zunächst im digitalen Geschäftsmodell (Synergie 5), dann im etablierten Geschäftsmodell (Synergie 6). Im Folgenden werden die sechs Synergien im Detail dargestellt und mithilfe von Beispielen erläutert. In der abschließenden Vignette verdeutlichen wir die verschiedenen Synergien anhand eines Fallunternehmens.

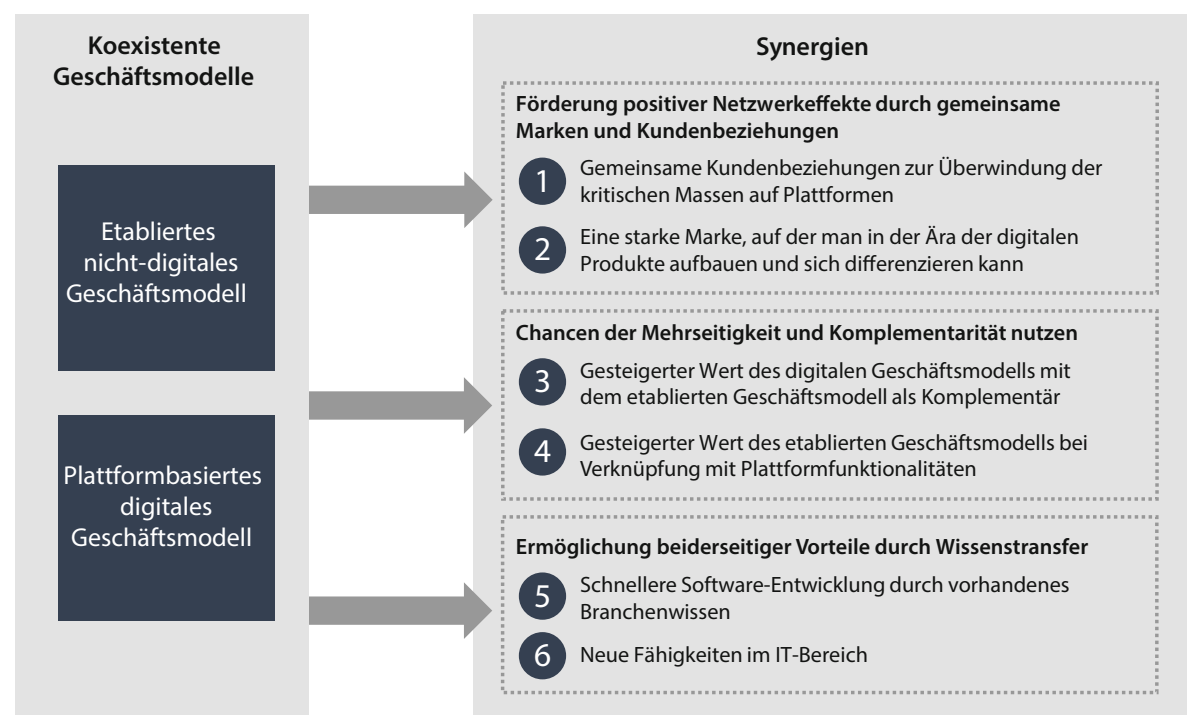

Abb. 1 Sechs Arten von Synergien zwischen koexistenten digitalen und etablierten Geschäftsmodellen 


\subsection{Gemeinsame Kundenbeziehungen zur Überwindung der kritischen Massen auf Plattformen (Synergie 1)}

Bestehende Vertriebskanäle, die auf das etablierte Geschäftsmodell spezialisiert sind, werden genutzt, um das neue digitale Geschäftsmodell zu fördern, sofern es ähnliche Kunden adressiert. Auf diese Weise können die vorhandenen Vertriebskräfte ein neues digitales Geschäftsmodell bei Kunden einführen, mit denen sie bereits regelmäßig zusammenarbeiten. Andere bestehende Kanäle, wie z.B. Industriemessen, können ebenfalls für das neue digitale Geschäftsmodell aktiviert werden. Die bestehenden Kundenbeziehungen sind nicht nur hilfreich, um Leads für zukünftige Einnahmen für das digitale Geschäftsmodell zu schaffen, sondern gewähren auch Zugang zu Kundenfeedback in einer frühen Entwicklungsphase. Der Neuaufbau solcher Kanäle und Beziehungen wäre deutlich teurer als die Wiederverwendung, sodass das digitale Geschäftsmodell von einer sub-additiven Kostensynergie profitiert. Während eine solche Synergie auch in der bisherigen Forschung beobachtet wurde, zwischen zwei parallelen nicht-digitalen Geschäftsmodellen, gewinnt sie im Zeitalter plattformbasierter digitaler Geschäftsmodelle eine völlig neue Bedeutung. Diese erste Synergie kann helfen, die notwendige kritische Masse an Konsumenten und Komplementären zu erreichen. Auch wenn nur eine Seite (z. B. nur Nutzer) erreicht werden, können die (seitenübergreifenden) Netzwerkeffekte die Attraktivität der Plattform weiter steigern.

Ein Beispiel für die vorteilhafte Nutzung gemeinsamer Kundenbeziehungen bietet Unternehmen 3 (UN3), welches in der Pharmabranche agiert. Das etablierte Geschäftsmodell des Unternehmens besteht in der Herstellung und dem Verkauf von Medikamenten zur Vorbeugung von Krankheiten für Menschen und Tiere. Es handelt sich hierbei um einen business-to-business (B2B) Ansatz. Das neue, digitale Geschäftsmodell von UN3 hingegen verfolgt einen business-to-consumer (B2C) Ansatz und verbindet Tierärzte mit Tierhaltern auf einer digitalen Plattform. Um schnell die kritische Masse an Tierärzten zu erreichen und die Plattform somit für Tierhalter attraktiv zu machen, nutzte UN3 seine bestehenden Vertriebskanäle und Kundenbeziehungen. Durch diesen vereinfachten Zugang kam das Pharmaunternehmen schnell mit der Hälfte aller Tierärzte auf dem Markt in Kontakt und konnte seine Plattform bewerben.

\subsection{Eine starke Marke, auf der man in der Ära der digitalen Produkte aufbauen und sich differenzieren kann (Synergie 2)}

Eine ähnliche Synergie besteht auch im Falle eines neuen digitalen Geschäftsmodells, das auf neue Kundensegmente abzielt. Das Wachstum des digitalen Geschäftsmodells kann dank der starken Marke, die im Laufe der Jahre durch das etablierte Geschäftsmodell aufgebaut wurde, noch beschleunigt werden. Eine starke Marke strahlt auch außerhalb des bestehenden Kundensegments und ist daher nützlich, um Vertrauen zu gewinnen und neue Kunden zu überzeugen. Diese super-additive Wertsynergie gewinnt in einem digitalen Umfeld aus mehreren Gründen zusätzlich an Bedeutung. Erstens können sich die Kunden nicht auf die physische Erfahrung (z.B. Berührungen) verlassen und benötigen daher andere Signale wie die Marke, 
um Vertrauen in ein digitales Geschäftsmodell zu gewinnen. Zweitens könnten sich Unternehmen in der gegenwärtigen Ära der Transparenz und des stets verfügbaren digitalen Wettbewerbs durch Preisvergleichs-Websites, Aggregatoren usw. schnell zu reinen Warenanbietern entwickeln und müssen sich kontinuierlich über ihre Marke differenzieren (Zealley et al. 2018). Weitere Netzwerkeffekte können dann den Erfolg der Plattform im Sinne von vielen Neukunden weiter vorantreiben.

Auch UN3 (siehe Abschn. 5.1) konnte das Wachstum seines digitalen Geschäftsmodells mit Hilfe der starken Marke des etablierten Geschäftsmodells beschleunigen. Die Marke (d.h. der deutsche Name von UN3) war hilfreich, um die Tierärzte schnell von der Seriosität des Vorhabens zu überzeugen und der digitalen Plattform beizutreten. Tierbesitzer waren im Gegensatz zu Tierärzten noch nicht näher mit der Marke vertraut, da diese im Rahmen der B2C-Strategie zum ersten Mal von UN3 direkt adressiert wurden. So wurde für die Tierbesitzer eine neue Marke gewählt, da der deutsche Name ungewohnt und schwer auszusprechen war.

\subsection{Gesteigerter Wert des digitalen Geschäftsmodells mit dem etablierten Geschäftsmodell als Komplementär (Synergie 3)}

Neue plattformbasierte digitale Geschäftsmodelle profitieren immens von einem bereits etablierten Geschäftsmodell, da es die Rolle eines Komplementärs übernehmen kann. Der Mehrwert der Plattform ist damit von Beginn an größer als ohne etabliertes Geschäftsmodell als Komplementär und es profitiert von einer super-additiven Wertsynergie: von Beginn an ist ein breiteres Angebot vorhanden, was das Vertrauen der Kunden steigert, sodass diese häufiger das kostenpflichtige PlattformAngebot wählen bzw. der Preis für dieses Angebot erhöht werden kann. In einem solchen Fall profitiert das Unternehmen von der Vielseitigkeit der Plattform und spielt gleichzeitig zwei Rollen, die des Plattformanbieters und die des Komplementärs. Auf diese Weise entsteht ein partielles Plattform-Ökosystem innerhalb eines einzigen Unternehmens.

Unternehmen 1 (UN1) ist traditionell in der Herstellung und dem Verkauf von Transportfahrzeugen tätig. Zusätzlich zu diesem etablierten Geschäftsmodell hat UN1 ein plattformbasiertes digitales Logistikmanagement entwickelt. Dieses neue digitale Geschäftsmodell basiert auf Telematikboxen, die von den Kunden in ihre Fahrzeuge eingebaut werden. In dieser Form ausgerüstete Fahrzeuge fungieren somit als Komplementäre. Das Unternehmen beschloss, alle selbst produzierten Fahrzeuge standardmäßig mit diesen Boxen auszurüsten, so dass jeder Kunde sofort eine Verbindung zur Plattform herstellen kann. Daher profitiert die Plattform frühzeitig von einer großen Anzahl von Komplementären.

\subsection{Gesteigerter Wert des etablierten Geschäftsmodells bei Verknüpfung mit Plattformfunktionalitäten (Synergie 4)}

Ein Plattform-Ökosystem innerhalb eines Unternehmens, wie im vorherigen Abschnitt gezeigt, ist auch für das etablierte Geschäftsmodell von Vorteil im Sinne einer super-additiven Wertsynergie. Etablierte Produkte oder Dienstleistungen, die den Kern etablierter Geschäftsmodelle bilden, sind direkt mit der Plattform ver- 
bunden und mit anderen Dienstleistungen auf der Plattform gekoppelt. Anstatt ein isoliertes etabliertes Produkt zu haben, wird das Produkt mit neuen Funktionen angereichert, die auf der Plattform angeboten werden, z. B. die Analyse von Daten, die aus den etablierten Produkten stammen. Dadurch steigert sich die Wertschöpfung im etablierten Geschäftsmodell und das etablierte Produkt bietet bei gleichem Preis nun einen Wettbewerbsvorteil, der zu höheren Umsätzen führen kann. Darüber hinaus stellt eine solche Plattform auch einen neuen Vertriebskanal für das etablierte Geschäftsmodell dar.

In dieser Form kann auch bei UN1 (siehe Abschn. 5.3) das etablierte Geschäftsmodell von dem neuen digitalen Geschäftsmodell profitieren. Die Fahrzeuge (Kern des etablierten Geschäftsmodells) sind um die digitalen Funktionen der Logistikmanagement-Plattform bereichert und weisen einen höheren Wert auf. Darüber hinaus können zwar Wettbewerber (z.B. andere Fahrzeughersteller) der Plattform beitreten, sobald die Kunden die Telematikboxen in ihren Fahrzeugen installiert haben, aber sie können sie nicht standardmäßig in ihre eigenen Lastwagen einbauen. Dadurch gewinnt das etablierte Geschäftsmodell von UN1 einen Wettbewerbsvorteil gegenüber seinen Konkurrenten.

\subsection{Schnellere Software-Entwicklung durch vorhandenes Branchenwissen (Synergie 5)}

Das neue digitale Geschäftsmodell kann das vorhandene Branchenwissen des etablierten Geschäftsmodells nutzen. Die sub-additive Kostensynergie besteht darin, dass dank des vorhandenen Fachwissens weniger Irrwege beschritten werden und die Entwicklungszeit verkürzt wird. Das Wissen über spezifische digitale Themen (z.B. Cloud-basierte Software-Entwicklung) mag gering sein, aber die Kenntnisse über die Branche, ihre Kunden und deren spezifische Schmerzpunkte sind tief. Daher ist der erste Schritt in der Software-Entwicklung, in dem es darum geht, einen Kunden zu verstehen und Schmerzpunkte vor der Entwicklung einer Lösung zu identifizieren (z. B. im Design Thinking-Prozess), schneller und somit kostengünstiger. Besonders in hochgradig regulierten Umgebungen wie dem Gesundheitswesen ist vorhandenes Branchen- und Regulierungswissen entscheidend. Etablierte Prozesse im Umgang mit sensiblen Gesundheitsdaten, zusammen mit einer erfahrenen und nahe gelegenen Rechtsabteilung, ermöglichen schnelle Anpassungen in Bezug auf den Datenschutz und eine schnellere Software-Entwicklung.

Ein Beispiel hierfür liefert Unternehmen 7 (UN7). Das etablierte Geschäftsmodell des Unternehmens besteht in der Herstellung dem Vertrieb hochwertiger Messund Medizintechnik. Mit einer digitalen Plattform zur Speicherung und Bearbeitung medizinischer Bilder und zur Durchführung automatisierter Analysen hat UN7 ein neues digitales Geschäftsmodell entwickelt. Der Schutz medizinischer Daten ist ein wichtiges und schwieriges Thema für dieses digitale Geschäftsmodell. Da das Unternehmen aber bereits umfassende Erfahrungen im Umgang mit medizinischen Daten und Prozessen mit Regulierungsbehörden aufgrund des etablierten Geschäftsmodells sammeln konnte, konnte es Datenschutzvorgaben und -probleme schnell angehen und lösen. 


\subsection{Neue Fähigkeiten im IT-Bereich (Synergie 6)}

Auch etablierte Geschäftsmodelle profitieren mittels einer sub-additiven Kostensynergie von digitalen Geschäftsmodellen. Neue digitale Geschäftsmodelle werden meist durch agile und cloudbasierte Softwareentwicklungspraktiken entwickelt, die für etablierte Unternehmen neu sind. So können insbesondere IT-MitarbeiterInnen durch die Zusammenarbeit mit ihren (in den meisten Fällen neu eingestellten) KollegInnen, die sich auf das neuartige digitale Geschäftsmodell konzentrieren, zusätzliche Fähigkeiten erwerben. Durch Methoden des Wissensaustauschs wie Job-Rotationen, Job-Shadowing, gemeinsame Workshops und Führungen werden die MitarbeiterInnen in neuen Kompetenzen geschult, zu deutlich geringeren Kosten als in extern durchgeführten Weiterbildungen. Diese neuen Kompetenzen werden genutzt, um bestehende Prozesse oder gar die IT-Architektur zu überarbeiten (z. B. höhere Produktivität durch mehr Release-Zyklen von Unternehmens-Anwendungen).

UN3 (siehe Abschn. 5.1) konnte sich die neuen IT Fähigkeiten ebenfalls zu Nutze machen. Einige IT-Manager des Unternehmens haben zwei Rollen inne und arbeiten sowohl in Bereichen für das etablierte Geschäftsmodell als auch in Bereichen für das neue digitale Geschäftsmodell. Dabei verbreiten sich Informationen über den neuartigen Technologie-Stack, der für die digitale Plattform verwendet wird. Dieser Technologie-Stack wird nun als Blaupause für die Modernisierung der etablierten IT-Architektur von UN3 verwendet.

Zusammenfassend profitiert vor allem das digitale Geschäftsmodell von Wertund Kostensynergien. Nichtsdestotrotz geht das etablierte Geschäftsmodell nicht leer aus. Die folgende Vignette eines Falles gibt beispielhaft alle sechs Synergien wieder. Sobald diese Synergien identifiziert sind, müssen sich Führungskräfte auf den schwierigen Weg machen, sie zu nutzen, wofür wir im nächsten Abschnitt Empfehlungen geben.

\section{Vignette: Synergien zwischen etabliertem und neuem digitalen Geschäftsmodell am Beispiel eines Unternehmens}

Das Unternehmen 7 in unserer Studie ist weltweit tätig auf dem Gebiet hochwertiger Instrumente für die Mess- oder Medizintechnik. Das Unternehmen verfolgt einen B2B-Ansatz im Sinne von Krankenhäusern, Forschungseinrichtungen und Industrieunternehmen als Endkunden.

Kürzlich hat das Unternehmen eine Bildanalyseplattform eingeführt und damit den Grundstein für ein neues plattformbasiertes digitales Geschäftsmodell gelegt. Ziel ist es, die Forschung zu demokratisieren, indem Analysen von medizinischen Bildern (z. B. von Mikroskopie-Aufnahmen) als Dienstleistung auf Pay-per-Use-Basis angeboten werden. Konsumenten können auf dieser Plattform Bilder hochladen, bearbeiten und automatisierte Analysen durchführen. Die Plattform ist offen für andere Medizintechnikfirmen (z. B. um Bilder aus ihren Produkten zu importieren) sowie für Startups (z. B. um zusätzliche Analyse-Algorithmen hinzuzufügen).

Bei der Entwicklung wurde der Datenschutz schon früh zu einem wichtigen Thema, da es sich um sensible medizinische Daten handelt. Aufgrund der um- 
fangreichen Erfahrung im Umgang mit Datenschutzfragen und -prozessen mit Regulierungsbehörden konnten offene Fragen zum Umgang mit solchen Daten in der Cloud schnell geklärt werden (Synergie 5), was als eine große Hürde für das digitale Geschäftsmodell angesehen wurde. Die Zeit bis zur Markteinführung wurde weiter verkürzt, da das Unternehmen seine großen Stände und seine bekannte Marke auf Branchenmessen nutzte, um schnell Feedback einzuholen (Synergien 1 und 2). Potenziellen Kunden, die sich zunächst über Innovationen der etablierten Produkte informieren wollten, wurden Prototypen der Plattform gezeigt. Ihr wertvolles Feedback verkürzte die Entwicklungszeit und senkte die Entwicklungskosten.

Wesentliche Komplementäre für die digitale Plattform sind Medizintechnikfirmen, die eine einfach zu bedienende Verbindung zwischen ihren Geräten und der Plattform ermöglichen. Zweifellos behindert eine komplexe Übertragung von Bildern von einem medizintechnischen Gerät (z. B. einem Mikroskop) auf die Plattform die Kundenzufriedenheit und schließlich den Erfolg der Plattform. Aus diesem Grund wurden die medizintechnischen Geräte, die den Kern des etablierten Geschäftsmodells bilden, schnell angepasst, um sie für eine einfache Bildübertragung zu optimieren. Von der Einführung des digitalen Geschäftsmodells an konnte sich die Plattform auf einen großen Komplementär stützen, was den Erfolg des Geschäftsmodells sichert (Synergie 3). Gleichzeitig bieten die medizintechnischen Geräte nun ab Auslieferung eine sehr enge Kopplung an die Plattform, somit auch neue Features und haben einen höheren Wert für die Endkunden (Synergie 4). Schließlich wurde intern darauf geachtet, dass regelmäßig Mitarbeiter des etablierten Geschäftsmodells in die Digital-Einheit rotieren. Das erleichterte die Anbindung an bestehende IT-Systeme, sorgte aber auch für eine Weiterbildung in Sachen Agile und Scrum bei ebendiesen Mitarbeitern, obwohl diese Digital-Einheit keinen originären Auftrag hatte zur Weiterbildung.

\section{Handlungsempfehlungen um Synergien zwischen koexistenten digitalen und nicht-digitalen Geschäftsmodellen wirksam einzusetzen}

Die tatsächliche Identifizierung und Nutzung von Synergien ist komplex (Nevo und Wade 2010) und hängt hauptsächlich vom Grad der Integration zwischen digitalen und etablierten Geschäftsmodellen ab. Daher könnten Führungskräfte versucht sein, beide Geschäftsmodelle eng miteinander zu verknüpfen. Eine solch enge Kopplung kann jedoch die Innovationsfähigkeit behindern. Sie kann Konflikte anheizen und das digitale Geschäftsmodell in Bezug auf die Entwicklungsgeschwindigkeit einschränken. Andererseits kann eine klare Trennung beider Geschäftsmodelle schnell zu Silodenken und Entfremdung führen und die Realisierung von Synergien unterbinden. Folglich müssen Unternehmen Integration und Trennung der Geschäftsmodelle ausbalancieren. Dazu empfehlen wir Maßnahmen entlang der Entwicklungsphasen eines digitalen Geschäftsmodells. Diese Maßnahmen ermöglichen einen großen Spielraum für Innovation in den frühen Phasen und die Nutzung von Synergien in den späte- 
ren Phasen der Entwicklung digitaler Geschäftsmodelle. Sie sind abgeleitet von den kumulativen Erfahrungen der untersuchten Unternehmen.

1. Bereits bei der Formulierung der Digital-Strategie des Unternehmens sollten die ersten Schritte unternommen werden, um den Weg für Synergien zwischen digitalen und nicht-digitalen Geschäftsmodellen zu ebnen. So könnte die Digital-Strategie die Vision enthalten, dass das digitale Geschäftsmodell auf dem etablierten Geschäftsmodell aufbaut und das etablierte Geschäftsmodell nicht isoliert, sondern durch das neue digitale Geschäftsmodell erweitert werden sollte, sodass beide Geschäftsmodelle zusammen ein Plattform-Ökosystem bilden, anstatt zwei vollständig getrennte Unternehmungen.

2. Unternehmen entscheiden sich oft dafür, eine neue „Digital“-Organisationseinheit an einem neuen Standort für ihr digitales Geschäftsmodell aufzubauen. Bei der Auswahl des neuen Standorts ist es wichtig, dass dieser noch in Reichweite des Hauptsitzes und der Einheiten liegt, die sich auf das etablierte Geschäftsmodell konzentrieren (z. B. weniger als eine Autostunde für die europäischen Fälle in unserer Studie). Eine solche Entfernung ermöglicht immer noch physische Begegnungen zwischen Mitarbeitern, was die Gefahr der Entfremdung oder des Silodenkens signifikant verringert. Weiterhin sollte die C-Ebene sicherstellen, dass das Managementteam, welches das digitale Geschäftsmodell operativ vorantreibt, aus internen Führungskräften mit engen Verbindungen zur übrigen Organisation besteht. Auf diese Weise kann das Managementteam als vertrauenswürdige Botschafter für das digitale Geschäftsmodell werben und die Botschaft verbreiten, dass digitale und nicht-digitale Geschäftsmodelle zusammenarbeiten und voneinander profitieren können.

3. Noch in der Entwicklung kann das digitale Geschäftsmodell von Synergien mit dem etablierten Geschäftsmodell profitieren und bestehende Kundenbeziehungen wiederverwenden (Synergie 1) als wichtige Quelle für Feedback. Wenn das digitale Geschäftsmodell auf ein völlig neues Kundensegment abzielt, kann es immer noch auf der etablierten Marke aufbauen, um schnell Partner oder potenzielle Kunden für ein solches Feedback zu sammeln (Synergie 2). Darüber hinaus kann das Team, welche das digitale Geschäftsmodell entwickelt, auf dem vorhandenen Branchenwissen und Kundenverständnis aufbauen, das im Rahmen des etablierten Geschäftsmodells vorhanden ist, um die Software-Entwicklung zu beschleunigen und so die Entwicklungskosten zu reduzieren (Synergie 5). Weitere Maßnahmen, die Führungskräfte auch auf niedrigeren Hierarchieebenen, ergreifen sollten, umfassen vorübergehende Jobrotation zwischen Organisationseinheiten, die an den koexistenten Geschäftsmodellen arbeiten, oder „Verbindungsleute“, d.h. Mitarbeiter, die sich auf ein Geschäftsmodell konzentrieren, werden physisch an den Arbeitsplatz der für das andere Geschäftsmodell verantwortlichen Einheit versetzt, während sie ihre ursprünglichen Aktivitäten beibehalten. Diese Bemühungen fördern die informelle Abstimmung zwischen den Mitarbeitern, ohne sie in ein formelles Format zu zwingen. In der Tat könnten formelle Abstimmungsmethoden wie z. B. Portfolio-Lenkungsausschüsse bei Mitarbeitern, die am digitalen Geschäftsmodell arbeiten, auf Widerstand stoßen, da sie befürchten könnten, gebremst zu werden. 
4. Um anschließend die notwendigen kritischen Massen bei plattformbasierten Geschäftsmodellen zu erreichen, können Führungskräfte bestehende Marketingund Vertriebsressourcen, Kanäle und Kundenbeziehungen neu ausrichten, um die neue digitale Plattform zu fördern. Selbst wenn sie möglicherweise nur eine Seite der Nutzer (z.B. Konsumenten oder Komplementäre) schnell ansprechen können, werden Netzwerkeffekte den Anreiz für die andere Seite steigern, sich der Plattform anzuschließen (Synergie 1). Auf der Grundlage desselben Prinzips der Netzwerkeffekte könnten etablierte Unternehmen in der Lage sein, ihr etabliertes Geschäftsmodell als Komplementär auf der Plattform zu platzieren. Als solcher befindet sich mindestens ein Komplementär von Anfang an auf der Plattform (Synergie 3). Eine Anpassung bestehender Produkte könnte notwendig sein und Führungskräfte sollten entsprechend handeln (z. B. Gewährleistung eines einfachen Datentransfers zwischen bestehendem Produkt und Plattform).

5. Mit einem skalierten digitalen Geschäftsmodell im Betrieb kann der Schwerpunkt auf das etablierte Geschäftsmodell verlagert werden. Sobald die etablierten Produkte mit der Plattform kompatibel sind, fungiert das etablierte Geschäftsmodell auf der Plattform als Komplementär und kann auf die gleiche Weise profitieren, wie andere Komplementäre: durch einen zusätzlichen Vertriebskanal. Darüber hinaus können Führungskräfte dank der Tatsache, dass sie beide Geschäftsmodelle im selben Unternehmen haben, beide Geschäftsmodelle so gestalten, dass die Verbindung zwischen der Plattform und dem etablierten Geschäftsmodell als Komplementär reibungsloser verläuft als bei anderen Komplementären, was zu einer besseren Kundenerfahrung und zu einem Wettbewerbsvorteil gegenüber anderen Komplementären, sprich Konkurrenten, führt (Synergie 4). Darüber hinaus kann das etablierte Geschäftsmodell in Bezug auf den Aufbau von Fähigkeiten profitieren (Synergie 6). Ein Wissenstransfer, der gemeinsame Workshops und Schulungen, gegenseitige Arbeitsplatzbesuche oder Jobrotationen umfassen kann, muss dafür eingerichtet werden. Die etablierte IT-Funktion ist besonders geeignet, von einem solchen Kompetenzaufbau zu profitieren, da sie neue Ansätze zur Softwareentwicklung, IT-Architektur und IT-Infrastruktur erlernen kann.

\section{Diskussion und Fazit}

Zusammenfassend lässt sich festhalten, dass viele etablierte Unternehmen im Rahmen ihrer digitalen Transformation auf digitale Geschäftsmodelle, insbesondere plattformbasierte, setzen. Was diese etablierten Unternehmen von Start-ups unterscheidet, die ähnliche digitale Geschäftsmodelle aufbauen, ist ihr bereits etabliertes Geschäftsmodell. Diese Studie deckt potenzielle Synergien zwischen koexistenten etablierten nicht-digitalen und neuen digitalen Geschäftsmodellen in Unternehmen auf und zeigt, dass beide Geschäftsmodelle voneinander profitieren können.

Während die Forschung bereits Erkenntnisse zu Synergien zwischen produktorientierten Geschäftsmodellen gewonnen hat (Wiener et al. 2018; Markides und Charitou 2004), bieten wir nun Einblicke in Synergien zwischen produktorientierten und digitalen plattformbasierten Geschäftsmodellen, welche ein inhärentes Thema der Wirtschaftsinformatik-Forschung darstellen. Insbesondere digitale plattformbasierte 
Geschäftsmodelle ermöglichen dank ihrer spezifischen Eigenschaften wie Mehrseitigkeit und Netzwerkeffekte neue Synergien zwischen Geschäftsmodellen. Wir zeigen, dass sowohl sub-additive Kostensynergien als auch super-additive Wertsynergien die Entwicklung und Skalierung des digitalen Geschäftsmodells beschleunigen und dem etablierten Geschäftsmodell zur Innovation verhelfen, sodass innerhalb eines einzigen Unternehmens ein Plattform-Ökosystem entstehen kann. Darüber hinaus bietet unsere Studie praktische Erkenntnisse für Führungskräfte. Ausgehend von unseren acht Fall-Unternehmen beschreiben wir detaillierte Maßnahmen, um Synergien zwischen den Geschäftsmodellen zu ermöglichen und zu heben.

Unsere Studie ist begrenzt in der Anzahl der untersuchten Fälle und Kulturen (alle Unternehmen haben ihren Hauptsitz in Deutschland). Darüber hinaus haben wir jeweils ausschließlich ein digitales und ein nicht-digitales Geschäftsmodell untersucht. Wir sind jedoch zuversichtlich, dass unsere Ergebnisse eine Grundlage schaffen, auf der zukünftige Forschung aufbauen kann. So gilt es, zusätzliche Interaktionen zwischen Geschäftsmodellen, z. B. Konflikte, besser zu verstehen und, insbesondere bei plattformbasierten Geschäftsmodellen, die Möglichkeit von mehr als zwei koexistenten Geschäftsmodellen zu untersuchen. Möglicherweise werden dabei auch Synergien zwischen Geschäftsmodell-Komponenten identifiziert, die die hier gefundenen Synergien nicht abgedeckt haben (z. B. Schlüsselaktivitäten) (Osterwalder und Pigneur 2010). In der Tat haben in einigen unserer Fälle die Unternehmen bereits beschlossen, weitere digitale Geschäftsmodelle zu entwickeln, auch als weitere Komplementäre zur ursprünglichen Plattform.

Funding Open Access funding enabled and organized by Projekt DEAL.

Open Access Dieser Artikel wird unter der Creative Commons Namensnennung 4.0 International Lizenz veröffentlicht, welche die Nutzung, Vervielfältigung, Bearbeitung, Verbreitung und Wiedergabe in jeglichem Medium und Format erlaubt, sofern Sie den/die ursprünglichen Autor(en) und die Quelle ordnungsgemäß nennen, einen Link zur Creative Commons Lizenz beifügen und angeben, ob Änderungen vorgenommen wurden.

Die in diesem Artikel enthaltenen Bilder und sonstiges Drittmaterial unterliegen ebenfalls der genannten Creative Commons Lizenz, sofern sich aus der Abbildungslegende nichts anderes ergibt. Sofern das betreffende Material nicht unter der genannten Creative Commons Lizenz steht und die betreffende Handlung nicht nach gesetzlichen Vorschriften erlaubt ist, ist für die oben aufgeführten Weiterverwendungen des Materials die Einwilligung des jeweiligen Rechteinhabers einzuholen.

Weitere Details zur Lizenz entnehmen Sie bitte der Lizenzinformation auf http://creativecommons.org/ licenses/by/4.0/deed.de. 


\section{Anhang}

Tab. 1 Details zu den analysierten Fall-Unternehmen

\begin{tabular}{|c|c|c|c|c|}
\hline Fall & $\begin{array}{l}\text { Etabliertes nicht-digitales } \\
\text { Geschäftsmodell } \\
\text { (B2B oder B2C) }\end{array}$ & $\begin{array}{l}\text { Digitales Geschäftsmodell } \\
\text { (B2B oder B2C) }\end{array}$ & $\begin{array}{l}\text { Kundensegment } \\
\text { des digitalen } \\
\text { Geschäfts- } \\
\text { modells }\end{array}$ & $\begin{array}{l}\text { Arten von } \\
\text { beob- } \\
\text { achteten } \\
\text { Synergien }^{\text {a }}\end{array}$ \\
\hline UN1 & $\begin{array}{l}\text { Herstellung und Verkauf von } \\
\text { Transportfahrzeugen (B2B) }\end{array}$ & $\begin{array}{l}\text { Plattformbasiertes digitales } \\
\text { Logistikmanagement (B2B) }\end{array}$ & Existierend & $3,4,5,6$ \\
\hline UN2 & $\begin{array}{l}\text { Herstellung und Verkauf von } \\
\text { Transportfahrzeugen (B2C) }\end{array}$ & $\begin{array}{l}\text { Online-Verkaufsplattform } \\
\text { für selten genutzte Fahrzeu- } \\
\text { ge (B2C) }\end{array}$ & Existierend & $3,4,5,6$ \\
\hline UN3 & $\begin{array}{l}\text { Herstellung und Verkauf von } \\
\text { Medikamenten zur Vorbeu- } \\
\text { gung von Krankheiten für } \\
\text { Mensch und Tier (B2B) }\end{array}$ & $\begin{array}{l}\text { Plattform, welche Tierärzte } \\
\text { und Tierhalter miteinander } \\
\text { verbindet (B2C) }\end{array}$ & $\begin{array}{l}\text { Existierend } \\
\text { (Tierärzte) } \\
\text { und neu } \\
\text { (Tierhalter) }\end{array}$ & $1,5,6$ \\
\hline UN4 & $\begin{array}{l}\text { Stationärer Verkauf von hoch- } \\
\text { wertiger Mode und Kosmetik } \\
\text { (B2C) }\end{array}$ & $\begin{array}{l}\text { Plattformbasierter Online- } \\
\text { Shop, der rund um die Uhr } \\
\text { hochwertige Mode und } \\
\text { Kosmetik verkauft (B2C) }\end{array}$ & Existierend & $\begin{array}{l}1,3,4,5, \\
6\end{array}$ \\
\hline UN5 & $\begin{array}{l}\text { Herstellung und Verkauf von } \\
\text { Komponenten und Systemen } \\
\text { für Fahrzeughersteller und } \\
\text { Ersatzteile für Logistikunter- } \\
\text { nehmen (B2B) }\end{array}$ & $\begin{array}{l}\text { Plattformbasierte Verfol- } \\
\text { gung des Frachtsystems, } \\
\text { integriert in das ERP-Sys- } \\
\text { tem (B2B) }\end{array}$ & $\mathrm{Neu}$ & $2,5,6$ \\
\hline UN6 & $\begin{array}{l}\text { Produktmanagement und sta- } \\
\text { tionärer Verkauf von Mobili- } \\
\text { tätsdienstleistungen (B2C) }\end{array}$ & $\begin{array}{l}\text { Online-Verkaufsplattform } \\
\text { für Mobilitätsdienstleistun- } \\
\text { gen (B2C) }\end{array}$ & Existierend & $\begin{array}{l}1,3,4,5, \\
6\end{array}$ \\
\hline UN7 & $\begin{array}{l}\text { Herstellung und Verkauf von } \\
\text { hochwertiger Mess- oder Me- } \\
\text { dizintechnik (B2B) }\end{array}$ & $\begin{array}{l}\text { Plattform zum Speichern } \\
\text { und Bearbeiten medizini- } \\
\text { scher Bilder und zur Durch- } \\
\text { führung automatisierter } \\
\text { Analysen (B2B) }\end{array}$ & $\begin{array}{l}\text { Existierend, } \\
\text { einige neu }\end{array}$ & $\begin{array}{l}1,2,3,4, \\
5,6\end{array}$ \\
\hline UN8 & $\begin{array}{l}\text { Erzeugung und Verkauf von } \\
\text { Energie an Privat- und Ge- } \\
\text { schäftskunden (B2B B2C) }\end{array}$ & $\begin{array}{l}\text { Intelligente Energie- und } \\
\text { Gebäudemanagement-Lö- } \\
\text { sungen als Software-as-a- } \\
\text { Service (B2B) }\end{array}$ & $\mathrm{Neu}$ & $1,2,5,6$ \\
\hline
\end{tabular}

$U N$ Unternehmen, $B 2 B$ business to business, $B 2 C$ business to consumer

a Synergie 1: Gemeinsame Kundenbeziehungen zur Überwindung der kritischen Massen auf Plattformen, Synergie 2: Eine starke Marke, auf der man im im Zeitalter digitaler Produkte aufbauen und über die man sich differenzieren kann,

Synergie 3: Gesteigerter Wert des digitalen Geschäftsmodells ergänzt durch das etablierte Geschäftsmodell,

Synergie 4: Gesteigerter Wert des etablierten Geschäftsmodells durch die Verbindung mit Plattformfunktionalitäten,

Synergie 5: Schnellere Software-Entwicklung durch vorhandenes Branchenwissen,

Synergie 6: Neue Fähigkeiten in der IT 


\section{Literatur}

Cennamo C, Santalo J (2013) Platform competition: strategic trade-offs in platform markets. Strat Manag J 34(11):1331-1350. https://doi.org/10.1002/smj.2066

Evans DS, Schmalensee R (2007) Catalyst code: the strategies behind the world's most dynamic companies. Business School Press, Harvard

Goold M, Campbell A (1998) Desperately seeking synergy. Harv Bus Rev 76(5):131-143

Hagiu A, Wright J (2015) Multi-sided platforms. Int J Ind Organ 43:162-174

Hanelt A, Busse S, Kolbe LM (2017) Driving business transformation toward sustainability: exploring the impact of supporting IS on the performance contribution of eco-innovations. Info Systems $\mathbf{J}$ 27(4):463-502. https://doi.org/10.1111/isj.12130

Hess T, Matt C, Benlian A, Wiesböck F (2016) Options for formulating a digital transformation strategy. MISQE 15(2):123-139

Hoßbach N (2015) Dual business models: going beyond spatial separation. Twenty-first American Conference on Information Systems (AMCIS), Puerto Rico

Katz ML, Shapiro C (1994) Systems competition and network effects. J Econ Perspect 8(2):93-115

Klein H, Myers MD (1999) A set of principles for conducting and evaluating interpretive field studies in information systems. MIS Q 23(1):67-94

Klostermeier R, Haag S, Benlian A (2018) Digitale Zwillinge - Eine explorative Fallstudie zur Untersuchung von Geschäftsmodellen. HMD. https://doi.org/10.1365/s40702-018-0406-x

Li L, Su F, Zhang W, Mao J-Y (2017) Digital transformation by SME entrepreneurs: a capability perspective. Info Systems J 28:1129-1157. https://doi.org/10.1111/isj.12153

Markides C, Charitou CD (2004) Competing with Dual Business Models: A Contingency Approach. Acad Manag Perspect 18(3):22-36

Martin JA, Eisenhardt KM (2001) Exploring cross-business synergies. Academy of Management Annual Meeting, Washington, D.C., USA

McIntyre DP, Srinivasan A (2017) Networks, platforms, and strategy: emerging views and next steps. Strat Manag J 38(1):141-160

McKinsey (2019) The right digital-platform strategy. In: Bughin J, Catlin T, Dietz M (Hrsg) McKinsey quarterly, Bd. 2019

Miles MB, Huberman AM (1994) Qualitative data analysis: an expanded sourcebook, 2. Aufl. SAGE, Thousand Oaks

Milgrom P, Roberts J (1995) Complementarities and fit strategy, structure, and organizational change in manufacturing. J Account Econ 19:179-208

Nevo S, Wade MR (2010) The formation and value of IT-enabled resources: antecedents and consequences of synergistic relationships. MIS Q 34(1):163-183

Ondrus J, Gannamaneni A, Lyytinen K (2015) The impact of openness on the market potential of multisided platforms: a case study of mobile payment platforms. J Inf Technol 30(3):260-275

Osterwalder A, Pigneur Y (2010) Business model generation: a handbook for visionaries, game changers, and challengers. John Wiley Sons Inc., Hoboken

Pflaum A, Schulz E (2018) Auf dem Weg zum digitalen Geschäftsmodell. HMD 55(2):234-251. https:// doi.org/10.1365/s40702-018-0401-2

Robins J, Wiersema MF (1995) A resource-based approach to the multibusiness firm: empirical analysis of portfolio interrelationships and corporate financial performance. Strat Manag J 16(4):277-299

Sebastian IM, Mocker M, Ross JW, Moloney KG, Beath C, Fonstad NO (2017) How big old companies navigate digital transformation. MISQE 16(3):197-213

Steininger DM (2018) Linking information systems and entrepreneurship: a review and agenda for ITassociated and digital entrepreneurship research. Info Systems J 29:363-407. https://doi.org/10.1111/ isj. 12206

Teece DJ (2010) Business models, business strategy and innovation. Long Range Plann 43(2-3):172-194. https://doi.org/10.1016/j.lrp.2009.07.003

Tiwana A (2014) Platform ecosystems aligning architecture, governance, and strategy. Elsevier, Amsterdam

Toutaoui J, Benlian A (2020) The whole is greater than the sum of its parts-Synergies between nondigital and digital business models within companies. 53rd Hawaii International Conference on System Sciences, Hawaii, USA

Veit D, Clemons E, Benlian A, Buxmann P, Hess T, Kundisch D, Leimeister JM, Loos P, Spann M (2014) Business models. Bus Inf Syst Eng 6(1):45-53. https://doi.org/10.1007/s12599-013-0308-y 
Wiener M, Hoßbach N, Saunders C (2018) Omnichannel businesses in the publishing and retailing industries: Synergies and tensions between coexisting online and offline business models. Decis Support Syst 109:15-26. https://doi.org/10.1016/j.dss.2018.01.008

Yin RK (2003) Case study research: design and methods. Applied Social Research Methods, Bd. 5. SAGE, Thousand Oaks

Zealley J, Wollan R, Bellin J (2018) Marketers need to stop focusing on loyalty and start thinking about relevance. Harv Bus Rev 21:1-8

Zott C, Amit R, Massa L (2011) The business model: recent developments and future research. J Manage 37(4):1019-1042. https://doi.org/10.1177/0149206311406265 